\title{
Exploring the limits of hepatic surgery for alveolar echinococcosis -10-years' experience in an endemic area of Austria
}

\author{
Stefanie Kuscher (iD · Irmgard Elisabeth Kronberger · Alexander Loizides · Michaela Plaikner • Marijana Ninkovic • \\ Andrea Brunner · Herbert Auer · Eva Maria Gassner · Dietmar Öfner · Stefan Schneeberger
}

Received: 9 April 2019 / Accepted: 15 April 2019 / Published online: 9 May 2019

(C) The Author(s) 2019

\begin{abstract}
Summary
Background Hepatic alveolar echinococcosis is a slow-growing, destructive parasitosis for which radical surgical resection is currently the only curative treatment. In case of complications by infiltrated vascular or biliary structures, interventional techniques are used. Pharmacotherapy is recommended postoperatively and for inoperability. The rare zoonotic occurs in the northern hemisphere and still poses a challenge in diagnostic and therapeutic management. Based in an endemic area in Austria, we evaluated a decade of surgical treatment for alveolar echinococcosis (AE) at our department.

Methods Clinical data of patients undergoing hepatic resection for $\mathrm{AE}$ at our department between 2005 and 2014 were collected. Every diagnosis was again verified by histology and PCR. The PNM staging of preoperative imaging was done in six-eye principle. This study was approved by the local Ethics Committee of
\end{abstract}

The authors Stefanie Kuscher and Irmgard Elisabeth Kronberger contributed equally to the manuscript.

S. Kuscher $(\bowtie) \cdot$ I. E. Kronberger · M. Ninkovic · D. Öfner · S. Schneeberger

Department of Visceral, Transplant and Thoracic Surgery,

Center of Operative Medicine, Medical University of Innsbruck, Anichstraße 35, 6020 Innsbruck, Austria

Stefanie.Kuscher@i-med.ac.at

A. Loizides · M. Plaikner · E. M. Gassner

Department of Radiology, Medical University of Innsbruck, Anichstraße 35, 6020 Innsbruck, Austria

\section{A. Brunner}

Institute of Pathology, Medical University of Innsbruck, Müllerstraße 44, 6020 Innsbruck, Austria

\section{H. Auer}

Department of Medical Parasitology, Institute of Specific Prophylaxis and Tropical Medicine, Medical University of Vienna, Kinderspitalgasse 15, 1090 Vienna, Austria the Medical University Innsbruck (registration number 20170307-1537).

Results Nine of 12 patients had an $\mathrm{R} 0$ resection. In one case, cure was achieved by a combination of surgery and radiofrequency ablation, one patient had R2 resection due to unexpected AE spread and another patient underwent salvage operation with lethal outcome. Three patients experienced early postoperative complications. Late postoperative complications included incisional hernia and cholestatic hepatopathy. 9 patients have been disease free for a mean period of 6 years.

Conclusion The relevance and perioperative risks of an extensive $\mathrm{AE}$ resection and avoidance of $\mathrm{R} 2$ resections are reflected in the present study. Determination of PNM stage and evaluation of in-all resectability build the foundation for successful surgical treatment even in an advanced AE stage.

Keywords Alveolar echinococcosis · Echinococcus multilocularis · Liver neoplasms · Parasitic liver diseases $\cdot$ Surgical procedures

\section{Main novel aspects of the following paper}

We could show that finding the diagnosis of alveolar echinococcosis remains a challenging task for clinicians despite modern imaging techniques. In our study, we included patients who underwent portal vein embolisation and extensive liver resection due to suspicion of malignant liver tumor but were diagnosed with alveolar echinococcosis in histological examination.

Especially in non-endemic areas, the PNM staging system is unpopular. We herein emphasize the importance of performing PNM staging in every case of alveolar echinococcosis. 


\section{Introduction}

The manifestation of an echinococcus multilocularis (EM) infection, known as alveolar echinococcosis (AE), represents a highly pathogenic and potentially deadly disease of visceral organs. The natural course consists of an asymptomatic incubation period of about 5-15 years, followed by a chronic period in which about one-third of the patients is diagnosed incidentally [1]. Preferentially, EM parasitizes the liver, mimicking hepatocellular or cholangiocellular carcinoma or carcinoma of the gall bladder, and progressively infiltrates the parenchyma and surrounding tissue, leading to up to $90 \%$ mortality rates within 10 years [2-4]. In contrast to the space-consuming cystic formation in the liver caused by Echinococcus granulosus, the prevalence of EM in the human liver is much less. EM is endemic mainly in the northern hemisphere with a wide distribution. Large numbers of $\mathrm{AE}$ cases are reported in China, representing more than $90 \%$ of the global cases [5]. In Central Europe, Austria represents one endemic area for AE. Between the years 1982 and 2002, 44 Austrian cases of AE were recorded at the Institute of Medical Parasitology at the Medical University of Vienna, 28 of whom were found in the western part of the country [6].

Patients get infected with EM by ingestion of fruit containing microscopically small eggs of the fox tapeworm or close contact to infected animals. Once the larvae have reached the human liver, they evolve into metacestodes forming alveolar hydatid cysts. The lesion usually appears as a spongy structure composed of numerous irregular small vesicles of several millimeters [3, 7, 8]. Walls of bile ducts and blood vessels may be invaded or obstructed, and also metastaticlike spread into other organs like lungs, spleen, heart, kidney, and brain in an advanced stage of the disease has been observed [8-11]. Advanced AE lesions can show central necrosis, appearing as a cavity that is sometimes inaccurately described as "cyst." In fact, it is not a cyst with an active germinal layer and hydatid fluid, but consists of semi-liquefied necrotic tissue penetrating the biliary tree. They are at a high risk of bacterial superinfection and can cause life-threatening sepsis [12]. Severe complications like cholangitis or liver abscess due to central necrosis of the parasitic lesion impair prognosis. Early and adequate diagnosis of an EM infection is one major factor to decrease mortality and morbidity rates [13]. Initial diagnosis is usually based on findings at radiologic imaging including conventional and advanced techniques of ultrasonography, computed tomography (CT), and magnetic resonance imaging (MRI), while serologic analyses and polymerase chain reaction (PCR) serve to confirm the diagnosis. Despite the available tools, diagnosis of $\mathrm{AE}$ remains a challenging task and several reports on (primarily) misdiagnosed cases have been made in recent years $[10,14,15]$.
Based on the well-established TNM (tumor, node, metastasis) staging system for malignant tumors, a clinical classification model for AE, designated as PNM (parasite lesion, neighboring organ invasion, metastatic spread) classification has been implemented. The PNM system is mainly based on imaging findings and was established to serve as a tool for international standardization of disease manifestation and description of EM spread, and to guide clinicians about the course of the disease [7, 16]. International guidelines are aged and current management of $\mathrm{AE}$ is based only on poor to moderate quality of evidence and recommendation strength [17].

Complete resection of the infiltrated tissue is the current therapy of choice. Percutaneous and endoscopic techniques have been reported as successful therapeutic alternatives especially in cases of biliary complications and for patients in whom surgical treatment is not possible [18]. Though still seen as controversial, liver transplantation (LT) has been performed as ultima ratio for unresectable advanced or hilar-infiltrating AE. Serious complications and relapses, however, remain substantial problems in LT patients [19-21]. Antihelminthic agents including albendazole and mebendazole are in use for inoperable patients, as well as for perioperative therapy for at least 2 years [14].

One of the first authors to describe liver surgery for $\mathrm{AE}$ was the Japanese surgeon Yoichi Kasai. His work dates back to the late sixties, a time before modern imaging techniques and chemotherapeutics for echinococcosis became routinely available [22]. Bresson-Hadni et al. reported their experience with 117 consecutive patients diagnosed with AE between 1972 and 1993 and found that advancements in diagnosis and treatment over that time period resulted in higher incidence and prevalence rates due to earlier detectability in an asymptomatic stage of disease. $\mathrm{Cu}-$ rative resection was achieved more routinely while radiological interventions increasingly replaced surgery in the palliative setting. Since the introduction of benzimidazole therapy in the late eighties, stabilization of EM lesions and decrease of complications including jaundice, bleeding, and portal hypertension were generally observed. With those achievements, survival rates increased significantly. Large cohort studies describing the outcome of surgical AE treatment are rare and often outdated. With the single-center study described here, we share our experience in 12 patients treated surgically over a 10-year period in an endemic area in Central Europe. Data analyses describe diagnosis, treatment, and outcome of patients surgically treated for hepatic AE.

\section{Methods}

The study was done in an observational retrospective design for which the approval for realization of the study was given by the Ethical Committee at the 
Medical University of Innsbruck (registration number: 20170307-1537). The mean postoperative followup time was $71(0-139)$ months. Electronic patient datasheets were scanned for discharge coding and for surgery coding indicative for echinococcosis from 2005 to 2014. Medical records including imaging findings, serological tests, surgical reports, pathologic findings, and discharge letters were reviewed. Hematoxylin and eosin (HE-)stained tissue samples of the specimens were re-examined by a local pathologist. Furthermore, paraffin-embedded specimens were sent to the national competence center for parasitological infections for PCR analysis. Patients were only included in this trial if the diagnosis was confirmed histologically and by PCR. AE staging according to the PNM classification system [16] was performed, following a six-eye principle, in consensus of three radiologists with expertise in abdominal CT and MRI diagnosis. Further detailed medical information of all study patients was then collected and entered into an anonymized computerized data file.

\section{Results}

Between 01/01/2005 and 31/12/2014 a total of 12 patients ( 6 women and 6 men) were treated surgically for histologically and PCR-proven hepatic AE at our institution. Mean patient age at time of surgery was 53 (34-80) years. Females were younger than males with 49 (34-61) years vs. 58 (34-80) years, respectively.

10 patients had a positive preoperative serologic test for EM, in 2 patients no test was done for initial misdiagnosis of cholangiocellular carcinoma. Preoperative CT showed intralesional calcifications as typical features in 10 out of 12 patients. A detailed history of all relevant comorbidities and preoperative baseline characteristics are shown in Table 1. A curative approach was intended in 11 patients, one patient underwent a salvage operation for complicated AE. Preoperative treatment with benzimidazole was prescribed in 9 patients, 2 did not receive pharma-

Table 1 Baseline characteristics of patients

\begin{tabular}{|c|c|}
\hline Baseline characteristics of AE patients & Total $(n=12)$ \\
\hline Age (years) & $53(34-80)$ \\
\hline Male/female (n) & $6 / 6$ \\
\hline CHD/arterial fibrillation $(n)$ & $3 / 2$ \\
\hline DM/arterial hypertension $(n)$ & $1 / 1$ \\
\hline Chronic renal failure $(n)$ & 1 \\
\hline Deep venous thrombosis/pulmonary artery embolism $(n)$ & $2 / 1$ \\
\hline Preoperative positive serologic test for EM (n) & 10 \\
\hline $\begin{array}{l}\text { Intralesional calcification in preoperative CT }(n=12) \\
\text { or/and } M R I(n=7) ; n\end{array}$ & 10 \\
\hline Preoperative ERC/portal vein embolization (n) & $3 / 2$ \\
\hline Preoperative benzimidazole therapy (n) & 9 \\
\hline
\end{tabular}

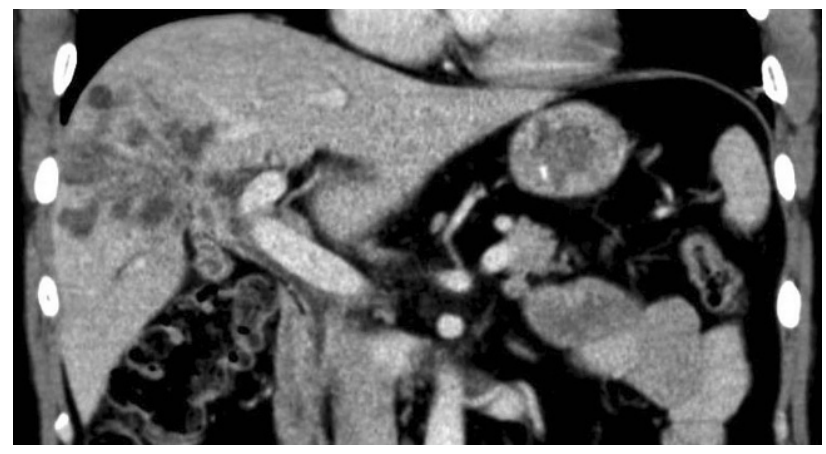

Fig. 1 CT scans show the extensive central hepatic alveolar echinococcosis (AE) infiltration of a patient, in whom the radiomorphological presentation of the lesions were misinterpreted as central cholangiocellular carcinoma

cotherapy due to the suspicion of carcinoma, and in one case, data could not be acquired in retrospect. The duration of preoperative pharmacotherapy was 4.7 months (mean 2-9 months). Two patients were misdiagnosed with carcinoma (Fig. 1), had portal vein embolisation for right liver augmentation, and underwent endoscopic retrograde cholangiography (ERC) and bile duct stenting before right extended hemihepatectomy $(\mathrm{HH})$ could be realized. One patient suffering from complicated AE had ERC and bile duct lavage with N-chlorotaurine (NCT) in consideration of a palliative salvage procedure.

With regard to the location of the parasitic mass in the liver, 4 cases were determined as P1, 5 cases as P3, and 3 cases showed a P4 status according PNM classification [16]. Extrahepatic involvement of neighboring organs and hence a positive $\mathrm{N}$ status was seen in 5 of our 12 patients. N1 stages included AE fistulation to the abdominal wall and suspected infiltration of regional lymph nodes in 4 cases. Two AE lesions were classified as M1 since metastatic spread to a para-aortic lymph node in the ventral mediastinum and to the right adrenal gland (without contact to the primary lesion) was suspected. Our data showed a PNM stage I in 2 cases, stage IIIa in 4 cases, stage IIIb in 2 cases, and stage IV in 4 cases. PNM classification and staging of patients are shown in Table 2. All 12 patients had a laparotomy. Four patients (33\%) underwent atypical or segmental resection, including resection of the middle hepatic vein in one case. In 8 patients $(67 \%)$, a major liver resection, including right and left (extended) $\mathrm{HH}$ with or without additional atypical liver resection, was necessary. Detailed information about surgical techniques, histology, additional therapy, and remission status is given in Table 2 .

In patient nr. 11 (see Table 2), an interdisciplinary approach including surgery and radiofrequency ablation (RFA) was done in order to treat an AE distributed to both liver lobes and a suspected spread to the mediastinum. Intraoperative RFA of an AE lesion in the right liver lobe was performed subsequent to left $\mathrm{HH}$, wedge resection of two right peripheral lesions, and 
Table 2 PNM classification and staging of patients with alveolar echinococcosis (AE), details of surgery and histology, medical therapy, and recurrence

\begin{tabular}{|c|c|c|c|c|c|c|}
\hline $\mathrm{Nr}$ & PNM staging & Hepatic surgery & Additional surgery or intervention & Complication & $\begin{array}{l}\text { Medical } \\
\text { therapy }\end{array}$ & Recurrence \\
\hline 1 & I (P1NOM0) & Atypical IVb, V & - & No & $?$ & $?$ \\
\hline 2 & I (P1NOM0) & Right $\mathrm{HH}$ & - & No & No & No \\
\hline 3 & IIIla (P3NOM0) & Right $\mathrm{HH}$ & Right adrenalectomy & No & Yes & No \\
\hline 4 & IIla (P3NOM0) & Atypical III, Iva, VI, VII & Middle liver vein & Bile leak (Dindo Illa) & Yes & No \\
\hline 5 & IIla (P3NOM0) & Right $\mathrm{HH}$, extended & Portal vein (bifurcation, left) & No & Yes & No \\
\hline 6 & IIla (P3NOM0) & Right $\mathrm{HH}$ & Part of left hepatic arteria, portal vein & Bile leak (Dindo Illb) & Yes & No \\
\hline 7 & IIIb (P3N1M0) & Atypical IV, V, unroofing & Lavage: $40 \%$ glucose (peritoneal seeding, R2) & No & Yes & Yes \\
\hline 8 & IIllb (P4NOM0) & Right $\mathrm{HH}$ & - & Bile leak (Dindo IIla) & Yes & No \\
\hline 9 & IV (P4N1M0) & Right $\mathrm{HH}$, extended & Mors in tabula, R2 & $\begin{array}{l}\text { Fatal bleeding } \\
\text { (Dindo V) }\end{array}$ & - & - \\
\hline 10 & IV (P4N1M0) & Left HH, extended & Lavage: $40 \%$ glucose & No & Yes & No \\
\hline 11 & IV (P1N0M1) & Left HH+ atypical right & Anterior mediastinum + RFA & No & Yes & No \\
\hline 12 & IV (P1N1M1) & Atypical IV, V, VI & Right adrenalectomy & No & Yes & No \\
\hline
\end{tabular}

mediastinal lymphadenectomy (Fig. 2). A second RFA (percutaneous) procedure with ablation of three lesions on the remaining right lobe at one week after surgery was performed.

In 9 cases, R0 resection of the hepatic AE lesion, in 3 patients a R2 resection was done. One of latter underwent additional RFA a week after surgery to reach a disease-free status. One patient had a palliative salvage operation and in one other case, unexpected additional liver and peritoneal lesions were found intraoperatively and only limited resection was performed.

All cases preoperatively suspected as stage $\mathrm{N} 1$ and/or M1, except the case with fistulating AE, could not be verified since the histopathological examination did eventually not confirm echinococcus invasion in the respective tissue or region.

Early complications occurred in 4 patients. According to the Clavien-Dindo classification, two grade IIIa, one grade IIIb, and one grade $\mathrm{V}$ complication, mean-

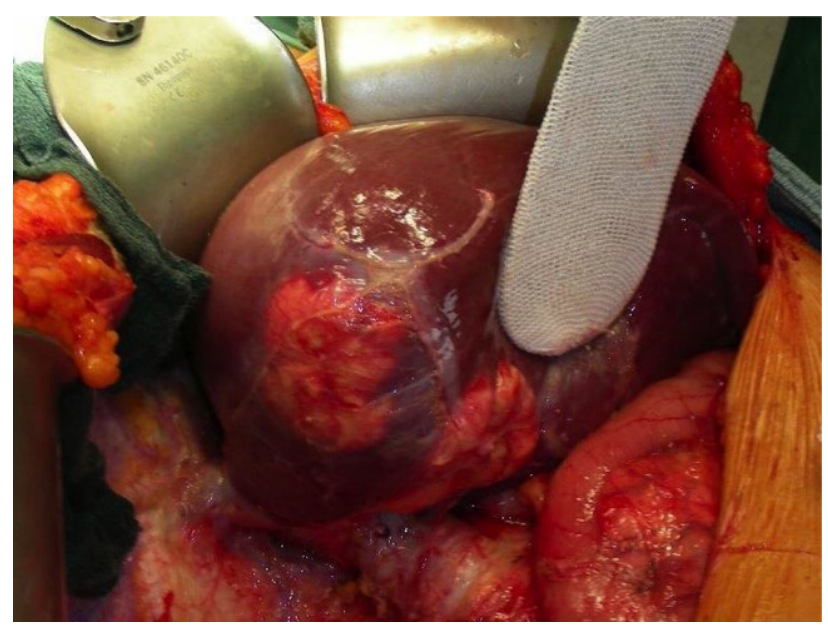

Fig. 2 Intraoperative presentation of $\mathrm{AE}$ liver infiltration in a radiological PNM stage IIla, P3NOMO ing intraoperative death of the patient, were recorded. In that latter case, profuse intraoperative bleeding was controlled with packing of the liver, the patient however died during surgery subsequent to a myocardial infarction. Two patients required drainage for a superinfected bilioma and accordingly an infected seroma, both on postoperative day 10. Relaparotomy for a bile leak on the first postoperative day was performed in one case.

As late complications there were incisional hernias 10 and 5 month after surgery and one cholestatic hepatopathy (without signs for AE relapse) 2 years after extended right $\mathrm{HH}$. Excluding the patient who died intraoperatively, mean postoperative intensive care stay was 1.2 days (0-4), mean overall hospital stay was 19 days (10-48).

Ten patients received postoperative pharmacotherapy with a mean duration of treatment of 10 months (3-24 months), one patient got lost to follow-up after discharge from our institution.

For 10 patients we could analyze, the follow-up was at least 14 months. In the 9 patients discharged with disease-free status, AE did not recur and the current mean disease-free survival is 87 months.

Disease progression 10 months postoperatively was seen in one case after $\mathrm{R} 2$ resection. Nineteen months after the surgery, this patient was reported as stable and without further progression of the lesions under benzimidazole treatment.

\section{Discussion}

The exposure to Echinococcus eggs is increased in human groups who spend more time in the wilderness and is highest in regions where sheep breeding is common [1]. In this study, the cause of acquisition of hepatic AE was unknown for the most part; however, one patient was a farmer and one had grown up on a farm. Another patient reported walking in the woods 
with the dog a lot and had seen warning signs for EM there. One patient had undertaken several journeys in Asia and experienced heavy diarrhea during his stay.

Our data once more illustrate the difficulty in the diagnosis and therapy of hepatic AE. Due to a resemblance of the radiologic features in liver imaging between cholangiocellular carcinoma and AE, misdiagnosis has been reported earlier as a pitfall for this disease $[2,10,23,24]$. In our study group, the initial indication for major liver resection was suspected intrahepatic cholangiocellular carcinoma in 2 out of $12 \mathrm{AE}$ patients. Serological prove of EM infection includes enzyme-linked immunosorbent assay (ELISA) and immunoblotting; however, discrimination between active and inactive lesions cannot always be made. Positive results can persist for years after spontaneous or benzimidazole-induced dying out of the metacestode, while the test is negative after surgical resection. In our small study group, alternating ELISA results at different timepoints, possibly resulting from the individual immunological status or benzimidazole intake, did not allow conclusive interpretation of our data. EM infection was indicated preoperatively in 10 cases, whether by species-specific ELISA and/or western blot. EM DNA can be detected by PCR, and reverse transcription PCR has been in use for the assessment of parasite viability. Good positive predictive value has been reported for these methods; however, a negative test does not guarantee absence of parasite activity and EM in the liver [14]. Here, only patients again positively tested for EM on PCR in paraffin-embedded formalin-fixed tissue were included. Furthermore, all patients had an abdominal CT scan preoperatively and intralesional calcifications were present in 10 cases. Ultrasonography usually serves as the first diagnostic imaging tool when clinical condition or blood test point to liver disease, while modern multislice CT and diffusion-weighted MRI are gold standard for precise description of focal liver lesions and most important for the assessment of biliary duct involvement and infiltration of hepatic blood vessels. Calcifications are characteristic CT findings in hepatic AE $[25,26]$.

Patients are often diagnosed with $\mathrm{AE}$ in an advanced stage, probably due to late onset of rather unspecific symptoms such as a flu-like condition, right upper quadrant pain, weight loss, and jaundice [27]. In our study group an advanced PNM stage IIIa/b or IV-involving both liver lobes or extrahepatic structures-was detected in 10 of 12 patients. However, postoperative histological examination of all specimens revealed only one case of extrahepatic $\mathrm{AE}$ spread which had not been recorded preoperatively. An N1 stage could not be confirmed histologically in 3 of 5 suspected cases. Both cases of previously suspected extrahepatic metastases (M1) were not confirmed. In our study population, the overestimation of $\mathrm{N}$ and $\mathrm{M}$ status may have been related to misinterpretation of reactive lymphadenopathy and unspecific extrahepatic lesions. Overall, R0 resection could be achieved in 7 out of 10 patients with $\mathrm{AE}$ stage IIIa/b or IV. When compared with a corresponding dataset where R0 resection in PNM stage IIIa/b or IV was achieved in only 9 of 23 patients [28], these results seem reasonable. In the latter trial, 5 cases had determination of inoperability intraoperatively. We observed one patient with an intraoperative diagnosis of inoperability and a limited resection was performed. These findings further emphasize the difficulty in the preoperative determination of PNM status and operability.

Intraoperative or early postoperative complications occurred in $33 \%(4 / 12)$ of our cases and included one intraoperative death, which conforms with overall post-hepatectomy complication rates reported in the literature [29-34]. McKay et al. quoted an inhospital mortality rate of $6 \%$ and an overall complication rate of $46 \%$ following hepatic resection, with hemorrhage and massive blood loss occurring most frequently [30]. As shown by Tzeng and colleagues in 2014 , elderly people ( $>75$ years) show higher morbidity rates despite a lesser surgical complexity and are at a much greater risk for mortality after severe post-hepatectomy complications than younger patients [35]. Joliat et al. reported Clavien-Dindo grade IIIa-V complications following liver resection for $\mathrm{AE}(n=59)$ in 6 patients $(10 \%)$; however, the low morbidity rate probably attributes to the fact that half of the patients were classified as PNM stage I and II (26 and 3 patients), therefore undergoing less extensive liver resection. In our study, 3 of 4 patients who experienced early postoperative complications had undergone surgery to the extent of a right $\mathrm{HH}$ or more. Post-hepatectomy bile leakage depicts one well-known complication that involves the risk for infection and peritonitis and is associated with poor outcome [36, 37]. According to the recommendation for radiology-guided drainage of small bilioma or seroma coming along with mild or no clinical symptoms [38], two of our patients were managed with percutaneous drainage. Another patient had early relaparotomy for a symptomatic bile leak. The cause for the intraoperative fatal outcome after a heart attack in one patient is certainly related to intraoperative blood loss and mass transfusion requiring liver packing for bleeding control. The patient was 80 years old and anticoagulated for atrial fibrillation and pulmonary embolism. Due to complicated necrotizing and fistulating $\mathrm{AE}$, the patient had previously needed emergency laparotomy twice in a peripheral hospital and had undergone palliative ERC and NCT lavage with the aim of controlling biliary complications, however, with no success. The indication for salvage operation and bridging of anticoagulation was thoroughly discussed in an interdisciplinary team. The risk profile of the patient was most significant, but the salvage operation was eventually offered to the patient because of the severe course and manifes- 
tation of the disease. This case may underline the subtle potential for serious complications and a fast and lethal course of disease in cases of late-diagnosed non-resectable hepatic $\mathrm{AE}$.

Whenever feasible, radical resection of the entire infected liver parenchyma with a safe distance of ideally $2 \mathrm{~cm}$ should be performed, according to the recommendation of the expert consensus for the diagnosis and treatment of cystic and alveolar echinococcosis in humans [7]. Radical in-all resection and in one patient additional RFA of hepatic AE lesions resulted in a clinically disease-free status in 10 of 12 patients after 6 years. In one case, intraoperative AE distribution appeared more extensive than expected and R2 status was assumed at the end of the operation. The value of palliative or debulking resections in cases of non-curable extended $\mathrm{AE}$ has been addressed by several authors [39]. Nine of our patients received postoperative benzimidazole therapy. Kadry et al. observed a clear survival benefit in patients treated by curative resection (R0) followed by 2-year benzimidazole therapy compared with antihelminthic therapy alone. R1 resection resulted in a higher rate of disease progression than curative surgery and did not appear to offer any advantage over benzimidazole therapy alone regarding complication [40]. Buttenschoen et al. reported $\mathrm{R} 0$ resection in all patients with stage I or II, whereas only in 9 of the remaining 23 patients (20 patients had stage III or IV) was an R0 resection achieved. Patients with procedures judged to be curative were still alive at the time of publication in $2008,50 \%$ of patients treated under palliative objections had died from AE, liver failure, or associated complications [28]. Furthermore, a Swiss group emphasizes the significance of an $\mathrm{R} 0$ resection with regard to the risk of $\mathrm{AE}$ recurrence [41]. In a retrospective analysis from the endemic area of eastern Anatolia, curative resection was achieved in $53 \%$ of all 32 surgically treated patients. In 7 patients in whom a curative surgical intervention was considered impossible, a cholangiojejunostomy of the intrahepatic bile duct was performed to treat jaundice caused by obstruction of the bile duct. Seven patients underwent laparotomy to evaluate resectability or in response to an inefficient percutaneous drainage of the EM lesion cavity. One patient underwent a debulking operation but died as a consequence of postoperative liver failure. Four patients died during the perioperative course due to (intraoperative) bleeding, progressive disease, and bronchobiliary fistula [42].

Since non-surgical treatment modalities have evolved and long-term benzimidazole therapy has shown acceptable results, palliative operations should be avoided and are reserved for individual cases of complicated AE which cannot be managed otherwise [7, $31,39]$. One of our patients showed an advanced AE stage preoperatively but due to the young age and good general condition, a curative surgical approach was pursued. Despite all efforts, the surgery resulted in an R2 situation. Progression of the disease with a large hepatic mass confirmed the insufficient disease control. The course of this case emphasizes the limited value of palliative $\mathrm{AE}$ resection.

Interventional techniques including transhepatic bile or abscess drainage, intralesional drug instillation, as well as ERC with duct dilatation or stenting may allow control of advanced $\mathrm{AE}$ and associated complications [17, 18, 43, 44]. Graeter et al. reported that the onset of hepatobiliary complications in their study group occurred at 3.7 years following the first AE diagnosis compared to 15 years in a study published by Frei et al. The latter group recorded survival of only 3 years following onset of hepatobiliary complications, while Graeter et al. reported 8.8 years survival $[12,44]$. Benzimidazole therapy is mandatory in all AE patients. Stabilization of the AE liver lesion can be achieved in the majority of cases; however, a risk for superinfection of necrotic areas, cholangitis, and sepsis remains. In case of surgery, temporary postoperative drug administration is recommended for at least 2 years, while lifelong treatment is needed in cases of incurable $\mathrm{AE}[7,18,40]$. In the present case series, we report one patient showing rapid $\mathrm{AE}$ re-growth after incomplete resection. Under anti-parasitic pharmacotherapy, the patient achieved stable disease for 2 years without parasiterelated complications. In 2010, the expert consensus for the diagnosis and treatment of cystic and alveolar echinococcosis in humans did not recommend presurgical antihelminthic pharmacotherapy, except for liver transplantation candidates [7].

\section{Conclusion}

Due to the similarity of radiomorphological appearance as well as clinical symptoms of hepatic $\mathrm{AE}$ and malignant liver tumor, revealing the diagnosis of hepatic $\mathrm{AE}$ can be a challenging task. Once $\mathrm{AE}$ is suspected, assessment of PNM stage on high-quality imaging and thorough evaluation of operability are mandatory and might be helpful for further therapy decision. Our data reflect the importance of in-all resection of the lesion(s) including the affected surrounding tissue. Even in an advanced AE stage with infiltration of central and hilar structures, good results in terms of disease-free survival can be achieved by major hepatectomy, provided no infected tissue is left behind. Interventional methods like RFA can serve as complementary therapeutic options in order to achieve an R0 situation in individual cases. In case of an R1 or R2 resection, rapid AE relapse is presumable. Palliative long-term benzimidazole treatment can allow a stable course of disease for years, but the risk for severe parasite-associated complications remains and most often involves the bile ducts. We recommend allocation of $\mathrm{AE}$ patients to hospitals with high expertise in the management and therapy of hepatic diseases. The adoption of the PNM classification system as a matter of routine as well as the 
establishment of stage-specific therapeutic strategies is advisable, not least with respect to comparability between the studies of this rare disease.

Funding Open access funding provided by University of Innsbruck and Medical University of Innsbruck.

\section{Compliance with ethical guidelines}

Conflict of interest S. Schneeberger has received personal fees from Astellas, Chiesi, Novartis, MSD, Shire, and ITB. S. Schneeberger has received a grant from Chiesi. S. Schneeberger has received travel support from Astellas, Chiesi, and Novartis. S. Schneeberger is a member of the expert group of Astellas and Chiesi. None of the mentioned declarations of S. Schneeberger were related to the conduction of the present study. S. Kuscher, I.E. Kronberger, A. Loizides, M. Plaikner, M. Ninkovic, A. Brunner, H. Auer, E.M. Gassner, and D. Öfner declare that they have no competing interests.

Ethical standards All procedures performed in studies involving human participants were in accordance with the ethical standards of the institutional and/or national research committee and with the 1964 Helsinki declaration and its later amendments or comparable ethical standards. The approval for realization of the presented retrospective study was given by the Ethics Committee at the Medical University of Innsbruck (registration number 20170307-1537, date of approval: 14th February 2017). Informed consent: due to the type of study (retrospective analysis), informed consent of every single patient was not mandatory.

Open Access This article is distributed under the terms of the Creative Commons Attribution 4.0 International License (http://creativecommons.org/licenses/by/4.0/), which permits unrestricted use, distribution, and reproduction in any medium, provided you give appropriate credit to the original author(s) and the source, provide a link to the Creative Commons license, and indicate if changes were made.

\section{References}

1. McManus DP, Zhang W, Li J, Bartley PB. Echinococcosis. Lancet. 2003;362:1295-304.

2. Stojkovic M, Mickan C, Weber TF, Junghanss T. Pitfalls in diagnosis and treatment of alveolar echinococcosis: A sentinel case series. BMJ Open Gastroenterol. 2015;2:e36.

3. Atanasov G, Benckert C, Thelen A, Tappe D, Frosch M, Teichmann D, Barth TF, Wittekind C, Schubert S, Jonas S. Alveolar echinococcosis-spreading disease challenging clinicians: A case report and literature review. World J Gastroenterol. 2013;19:4257-61.

4. McManus DP, Li Z, Yang S, Gray DJ, Yang YR. Case studies emphasising the difficulties in the diagnosis and management of alveolar echinococcosis in rural China. Parasit Vectors. 2011;4:196.

5. Vuitton DA, Zhou H, Bresson-Hadni S, Wang Q, Piarroux M, Raoul F, Giraudoux P. Epidemiology of alveolar echinococcosis with particular reference to China and Europe. Parasitology. 2003;127(7):S85-S105.

6. Auer H. Relevance of parasitological examinations for the clinical course, epidemiology and prevention of alveolar echinococcosis-Experiences of more than two decades in Austria. Wien Klin Wochenschr. 2006;118:18-26.

7. Brunetti E, Kern P, Vuitton DA, Writing Panel for the WHOIWGE. Expert consensus for the diagnosis and treatment of cystic and alveolar echinococcosis in humans. Acta Trop. 2010;114(1):1-16.

8. Czermak BV, Akhan O, Hiemetzberger R, Zelger B, Vogel W, Jaschke W, Rieger M, Kim SY, Lim JH. Echinococcosis of the liver. Abdom Imaging. 2008;33:133-43.

9. WorldHealth Organization, FoodandAgricultureOrganization of the United Nations. Multicriteria-based ranking for risk management of food-borne parasites. In: World Health Organization, editor. Food and agriculture organization of the united nations. Microbiological risk assessment series, Vol. 23. Genf: WHO; 2014.

10. Bulakci M, Yilmaz E, Cengel F, GocmezA, Kartal MG, IsikEG, Celenk E, Yegen G, Salmaslioglu A. Disseminated alveolar hydatid disease resembling a metastatic malignancy: A diagnostic challenge-A report of two cases. Case Rep Radiol. 2014; https:// doi.org/10.1155/2014/638375.

11. Kantarci M, Bayraktutan U, Karabulut N, Aydinli B, Ogul H, Yuce I, Calik M, Eren S, Atamanalp SS, Oto A. Alveolar echinococcosis: Spectrum of findings at cross-sectional imaging. Radiographics. 2012;32:2053-70.

12. Frei P, Misselwitz B, Prakash MK, Schoepfer AM, Prinz Vavricka BM, Müllhaupt B, Fried M, Lehmann K, Ammann RW, Vavricka SR. Late biliary complications in human alveolar echinococcosis are associated with high mortality. World J Gastroenterol. 2014;20:5881-8.

13. WHO Informal Working Group on Echinococcosis. Guidelines for treatment of cystic and alveolar echinococcosis in humans. WHO Informal Working Group on Echinococcosis. Bull World Health Organ. 1996;74:231-42.

14. Nunnari G, Pinzone MR, Gruttadauria S, Celesia BM, Madeddu G, Malaguarnera G, PavoneP, Cappellani A, Cacopardo B. Hepatic echinococcosis: clinical and therapeutic aspects. World J Gastroenterol. 2012;18:1448-58.

15. Aoki T, Hagiwara M, Yabuki H, Ito A. Unique MRI findings for differentiation of an early stage of hepatic alveolar echinococcosis. BMJ Case Rep. 2015; https://doi.org/10. 1136/bcr-2014-208123.

16. Kern P, Wen H, Sato N, Vuitton DA, Gruener B, Shao Y, Delabrousse E, Kratzer W, Bresson-Hadni S. WHO classification of alveolar echinococcosis: Principles and application. Parasitol Int. 2006;55:S283-S7.

17. Tamarozzi F, Vuitton L, Brunetti E, Vuitton DA, Koch S. Nonsurgical and non-chemical attempts to treat echinococcosis: Do they work? Parasite. 2014;21:75.

18. Vuitton DA, Azizi A, Richou C, Vuitton L, Blagosklonov O, Delabrousse E, Mantion GA, Bresson-Hadni S. Current interventional strategy for the treatment of hepatic alveolar echinococcosis. Expert Rev Anti Infect Ther. 2016;14:1179-94.

19. Bresson-Hadni S, Blagosklonov O, Knapp J, Grenouillet F, Sako Y, Delabrousse E, Brientini MP, Richou C, Minello A, Antonino AT, et al. Should possible recurrence of disease contraindicate liver transplantation in patients with endstage alveolar echinococcosis? A 20-year follow-up study. Liver Transpl. 2011;17:855-65.

20. Ozdemir F, Ince V, Barut B, Onur A, Kayaalp C, Yilmaz S. Livingdonor liver transplantation for Echinococcus alveolaris: Single-center experience. Liver Transpl. 2015;21:1091-5.

21. Koch S, Bresson-Hadni S, Miguet JP, Crumbach JP, Gillet M, Mantion GA, Heyd B, Vuitton DA, Minello A, Kurtz $S$, Clinicians EC. Experience of liver transplantation for incurable alveolar echinococcosis: A 45-case European collaborative report. Transplantation. 2003;75:856-63.

22. Kasai Y. Surgical treatment of liver diseases. Masui. 1969;18:1193-8.

23. Amano T, Hayashi S, Nishida T, Matsubara T, Takahashi K, Nakamatsu D, Tomimaru Y, Yamamoto M, Nakajima S, 
Fukui K, et al. Alveolar Echinococcosis mimicking a hepatic neoplasm with lymph node metastasis: A case report. Case Rep Gastroenterol. 2018;12:587-96.

24. Caire Nail L, Rodríguez Reimundes E, Weibel Galluzzo C, Lebowitz D, Ibrahim YL, Lobrinus JA, Chappuis F. Disseminated alveolar echinococcosis resembling metastatic malignancy: A case report. J Med Case Rep. 2017;11:113.

25. Liu W, Delabrousse É, Blagosklonov O, Wang J, Zeng H, Jiang Y, Qin Y, Vuitton DA, Wen H. Innovation in hepatic alveolar echinococcosis imaging: Best use of old tools, and necessary evaluation of new ones. Parasite. 2014;21:74.

26. Mueller J, Stojkovic M, Berger AK, Rosenberger KD, Schlett CL, Kauczor HU, Junghanss T, Weber TF. How to not miss alveolar echinococcosis in hepatic lesions suspicious for cholangiocellular carcinoma. Abdom Radiol (NY). 2016;41:221-30.

27. Piarroux M, Piarroux R, Giorgi R, Knapp J, Bardonnet K, Sudre B, Watelet J, Dumortier J, Gérard A, Beytout J, et al. Clinical features and evolution of alveolar echinococcosis in France from 1982 to 2007: Results of a survey in 387 patients. J Hepatol. 2011;55:1025-33.

28. Buttenschoen K, Carli Buttenschoen D, Gruener B, Kern P, Beger HG, Henne-Bruns D, Reuter S. Long-term experience on surgical treatment of alveolar echinococcosis. Langenbecks Arch Surg. 2009;394:689-98.

29. Cescon M, Vetrone G, Grazi GL, Ramacciato G, Ercolani G, Ravaioli M, Del Gaudio M, Pinna AD. Trends in perioperative outcome after hepatic resection: Analysis of 1500 consecutive unselected cases over 20 years. Ann Surg. 2009;249:995-1002.

30. McKayA, You I, Bigam D, Lafreniere R, SutherlandF, GhaliW, Dixon E. Impact of surgeon training on outcomes after resective hepatic surgery. Ann Surg Oncol. 2008;15:1348-55.

31. Du C, Liu Z, Yang X, Yan L, Li B, Wen T, Yang J, Xu M, Chen Z, Wang W. Hepatectomy for patients with alveolar echinococcosis: Long-term follow-up observations of 144 cases. IntJSurg. 2016;35:147-52.

32. Kayaalp C, Bzeizi K, Demirbag AE, Akoglu M. Biliary complications after hydatid liver surgery: Incidence and risk factors. J Gastrointest Surg. 2002;6:706-12.

33. Braunwarth E, Stättner S, Fodor M, Cardini B, Resch T, Oberhuber R, Putzer D, Bale R, Maglione M, Margreiter C, et al. Surgical techniques and strategies for the treatment of primary liver tumours: Hepatocellular and cholangiocellular carcinoma. Eur Surg. 2018;50:100-12.
34. Pereyra D, Starlinger P. Shaping the future of liver surgery: Implementation of experimental insights into liver regeneration. Eur Surg. 2018;50:132-6.

35. Tzeng CW, Cooper AB, Vauthey JN, Curley SA, Aloia TA. Predictors of morbidity and mortality after hepatectomy in elderly patients: Analysis of 7621 NSQIP patients. HPB (Oxford). 2014;16:459-68.

36. Yamashita Y, Hamatsu T, Rikimaru T, Tanaka S, Shirabe K, Shimada M, Sugimachi K. Bile leakage after hepatic resection. Ann Surg. 2001;233:45-50.

37. Shen S, Kong J, Qiu Y, Zhang S, Qin Y, Wang W. Ex vivo liver resection and autotransplantation versus allotransplantation for end-stage hepatic alveolar echinococcosis. Int J InfectDis. 2018;79:87-93.

38. Jin S, Fu Q, Wuyun G, Wuyun T. Management of posthepatectomy complications. World J Gastroenterol. 2013;19:7983-91.

39. Buttenschoen K, Gruener B, Carli Buttenschoen D, Reuter S, Henne-Bruns D, Kern P. Palliative operation for the treatment of alveolar echinococcosis. Langenbecks Arch Surg. 2009;394:199-204.

40. Kadry Z, Renner EC, Bachmann LM, Attigah N, Renner EL, Ammann RW, Clavien PA. Evaluation of treatment and long-term follow-up in patients with hepatic alveolar echinococcosis. BrJSurg. 2005;92:1110-6.

41. Joliat GR, Melloul E, Petermann D, Demartines N, Gillet M, Uldry E, Halkic N. Outcomes after liver resection for hepatic alveolar echinococcosis: A single-center cohort study. World J Surg. 2015;39:2529-34.

42. Emre A, Ozden I, Bilge O, Arici C, Alper A, Okten A, Acunas B, Rozanes I, Acarli K, Tekant Y, Ariogul O. Alveolar echinococcosis in Turkey. Experience from an endemic region. Dig Surg. 2003;20:301-5.

43. Stojkovic M, Junghanss T, Veeser M, WeberTF, Sauer P.Endoscopic treatment of biliary stenosis in patients with alveolar echinococcosis-Report of 7 consecutive patients with serialERC approach. Plos Negl Trop Dis. 2016;10:e4278.

44. Graeter T, Ehing F, Oeztuerk S, Mason RA, Haenle MM, Kratzer W, Seufferlein T, Gruener B. Hepatobiliary complications of alveolar echinococcosis: A long-term follow-up study. World J Gastroenterol. 2015;21:4925-32.

Publisher's Note Springer Nature remains neutral with regard to jurisdictional claims in published maps and institutional affiliations. 